\title{
TREES AND ISOLS, PART I
}

\section{T. G. MCLAUGHLIN}

1. Introduction. Let $N$ denote the set $\{0,1,2,3, \cdots\}$ of all natural numbers. By a tree we shall here mean the graph of a partial numbertheoretic function $f$ having the properties: (1) $\rho f \subseteq \delta f$, where $\rho f$ denotes the range of $f$ and $\delta f$ denotes the domain of $f$; and (2) $(\forall x) \quad\left[x \in \rho f \Rightarrow(\exists y)\left(f^{y}(x)=f^{y+1}(x)\right)\right]$, where we define $f^{0}(x)$ $=x$ and $f^{n+1}(x)=f\left(f^{n}(x)\right)$ for all $n \in N$ and all $x \in \delta f$. If $f^{-1}(x)$ is finite for all $x \in \rho f$, we say that the tree is finite-branching. The reader should note that other authors generally use the term tree in senses slightly different from the above. We shall say that a tree is semicomputable if it is the graph of a partial recursive function $f$ having properties (1) and (2). (Thus, we call a tree semicomputable in place of calling it recursively enumerable.) If $f$ is a partial numbertheoretic function with properties (1) and (2) and if $T$ is the graph of $f$, then $T$ is said to be a non-trivial tree just in case $T$ has at least one infinite branch; i.e., $T$ is non-trivial provided there exists a one-to-one function $g: N \rightarrow N$ such that $f(g(0))=g(0) \&(\forall n)[f(g(n+1))=$ $g(n)]$. (We shall usually identify a branch of $T$ with the set of "nodes" occurring along it; i.e., with $\rho g$ where $g$ is a one-to-one function such that $\{\langle g(n), f(g(n))\rangle \mid n \in \delta g\}=$ the branch in question.) Thus, a non-trivial semicomputable tree is the graph of what has elsewhere been called a special regressing function (see, for example, [1] ).

By a $\Delta_{2}$ function we mean a function $f: N \rightarrow N$ such that the graph of $f$ is explicitly definable in both 2-quantifier forms in the arithmetical hierarchy; equivalently, in view of the Kleene-Post theorem, $f$ is $\Delta_{2} \Leftrightarrow f$ is recursive in the degree $0^{1}$ of the halting problem. Let $T_{0}$ and $T_{1}$ be trees, corresponding respectively to partial number-theoretic functions $f_{0}$ and $f_{1}$; then we say that $T_{0}$ is a $T_{1}$ skeleton, and write $T_{0}-3 T_{1}$, provided that (a) if $\alpha$ is any infinite set regressed by $f_{1}$ then $\alpha$ has exactly one infinite subset $\beta$ regressed by $f_{0}$, and (b) $\delta f_{0} \subseteq \delta f_{1} \quad \& \quad(\forall x)\left[x \in \delta f_{0} \Rightarrow f_{0}(x) \in \hat{f}_{1}(x)\right]$, where $f_{1}(x)=\left\{x, f_{1}(x), f_{1}\left(f_{1}(x)\right), \cdots\right\}$. (In the context of this definition, the words "regressed by $f_{1}$ " do not carry the requirement that $f_{1}$ be partial recursive. Thus, here, " $\alpha$ is an infinite set regressed by $f_{1}$ " simply means that there is a one-to-one function $g: N \rightarrow N$ such that $\rho g=\alpha \& f_{1}(g(0))=g(0) \&(\forall n)\left[f_{1}(g(n+1))=g(n)\right], \quad$ and similarly for $f_{0}$ and $\beta$.) If , given $T_{0}-3 T_{1}$, there is a fixed recursively enumerable set $\gamma$ such that in (a) we always have $\alpha-\beta=\alpha \cap \gamma$, then

Received by the Editors October 5, 1973 and in revised form April 19, 1974. 
we say that $T_{0}$ is a uniformly co-enumerable $T_{1}$-skeleton, and we write $T_{0}-_{+} T_{1}$. (Naturally, we are interested in the relations $T_{0}-3 T_{1}, T_{0}$ $-3+T_{1}$ only in case $T_{1}$ is non-trivial.) Clearly, both -3 and $-3_{+}$are transitive relations. Let $T$ be a tree determined by a partial numbertheoretic function $f$, and let a function $g: N \rightarrow N$ be given. We shall say that $T$ is g-dominant $\Leftrightarrow{ }_{d f}(\forall n)[(n \in \delta f \& f(n) \neq n \& n$ lies on at least one infinite branch of $T) \Rightarrow n>g(f(n))]$.

Our basic result is that for every non-trivial semicomputable tree $T$ and every $\Delta_{2}$ function $g$ there exists a $g$-dominant semicomputable tree $T_{\mathrm{g}}$ (finite-branching if $T$ is finite-branching) such that $T_{\mathrm{g}}-3+T$. Various applications to the theory of the regressive isols are exhibited in §3; in particular, the main theorem of [14] is derived as a rather easy corollary. All unexplained (and non-self-explanatory) special notation and terminology appearing in $\$ \$ 2$ and 3 should be read in accordance with the conventions of $[13, \$ 1]$, with the following exceptions: the notations $\varphi_{e}$ and $\varphi_{e}^{s}$ of [13] are here replaced by $\varphi_{e}{ }^{1}$ and $\varphi_{e}{ }^{1, s}$ respectively, this change being made to eliminate a minor ambiguity from the notational scheme of [13]; also, we stipulate $\varphi_{0}{ }^{1}=$ the identity function.

In addition to the applications to be considered in $\$ 3$ of this paper, there are a number of applications (to be presented in a subsequent article) to the class of universal regressive isols. These isols were first studied by Ellentuck, in some unpublished notes circulated by him around 1968. Ellentuck has since obtained a variety of interesting results about such isols, including some which have appeared in [11]. We conclude this introduction by mentioning a theorem which can, as we shall demonstrate in our later article, rather easily be derived with the aid of the procedures used in proving Theorem 2.2 below: let a be a recursively enumerable degree such that $\mathbf{a}^{1}=0^{11}$, and let $\langle P, \leqq\rangle$ be a countable partial order; then there exists a recursively enumerable family $u$ of co-simple universal regressive isols, each of degree a, such that (i) the closure of $U$ under finite sums consists entirely of regressive isols and (ii) $\langle P, \leqq\rangle$ can be orderimbedded into $U$. Indeed, this can be proven in such a way as to answer, at the same time, a question about $\leqq_{\Lambda^{-}},<_{\Lambda^{-}}$embeddings indicated on p. 636 of [11]. Ellentuck's work on universal isols contains many other interesting results less closely related to the methodology of the present paper.

2. The basic theorem: existence of g-dominant, semicomputable, uniformly co-enumerable $T$-skeletons, for all $g \in \Delta_{2}$.

2.1. Lemma (Shoenfield, [18]). If $g$ is a $\Delta_{2}$ function then there exists a recursive function $g_{0}: N \times N \rightarrow N$ such that $(\forall x)\left[\lim _{y \rightarrow \infty} g_{0}\right.$ $(x, y)$ exists and is equal to $g(x)]$. 
2.2. Theorem. Let $g$ be a $\Delta_{2}$ function, and let $T_{1}$ be a non-trivial semicomputable tree. Then there exists a g-dominant semicomputable tree $T_{0}$ such that $T_{0}-3_{+} T_{1}$. Moreover, $T_{0}$ is finite-branching if $T_{1}$ is.

Proof. Let $g_{0}$, a recursive function of two variables, be related to $g$ as in Lemma 2.1. In all that follows, the notations $f^{*}(x)$ and $\hat{f}(x)$ (for an arbitrary partial number-theoretic function $f$ satisfying (1) and (2) of $\$ 1$, and for an arbitrary $x \in \delta f$ ) shall have the meanings assigned them in $[13, \S 1]$. Let $f_{1}$ be the partial recursive function to which $T_{1}$ corresponds as graph. We shall assume, for convenience, that $0 \notin \delta f_{1}$; this entails no genuine loss of generality. Since $T_{1}$ is semicomputable (i.e., since $f_{1}$ is partial recursive), there exists a recursive function $\hat{h}$ such that $(\forall n)\left[\delta \varphi_{\tilde{h}(n)}^{1}=\left\{y \mid y \in \delta f_{1} \& f_{1}^{*}(y)\right.\right.$ $=n\}]$; that is to say, the "levels" of $T_{1}$ form a recursively enumerable sequence of pairwise-disjoint r.e. sets. We shall employ an infinite array $\left\langle\Lambda_{i j}\right\rangle_{i=0, j=0}^{\infty}$ of approximation markers, an infinite array $\left\langle\Sigma_{i j}\right\rangle_{i=0, j=0}^{\infty}$ of reservation tags, and (an unlimited supply of copies of) a special barrier marker $\mathcal{B}$. The markers $\Lambda_{i j}$ are given an (sequential) ordering by the rule that $\Lambda_{k \ell}$ has higher priority than $\Lambda_{m n} \Leftrightarrow d f \pi_{2}(k, l)<\pi_{2}(m, n)$, where $\pi_{2}$ is a fixed one-to-one recursive function from $N \times N$ onto $N$; for reasons of technical convenience, we stipulate that $\pi_{2}(0,0)=0$ and that $\pi_{2}$ is increasing separately in each of its variables. Here and throughout the remainder of the paper, we shall denote by $W_{n}$ the set $\delta \varphi_{n}{ }^{1}, n \in N$. Thus $W_{n}$ is the $n$-th recursively enumerable subset of $N$, in a standard enumeration of the class of all such subsets. Let $k$ be a two-place recursive function such that $(\forall n)\left[\rho \hat{k}_{n}={ }_{d f} \rho \hat{k}(n, y)=W_{\tilde{h}(n)}\right]$. We are now ready to describe the stage-by-stage construction by means of which $T_{0}$ is to be obtained from $T_{1}$. In this construction, we shall define $T_{0}$ by stages as the graph of a partial recursive function $p_{0}$ having the property that $\delta p_{0} \subseteq \delta f_{1} \&(\forall x)\left[x \in \delta p_{0} \Rightarrow p_{0}(x) \in \hat{f}_{1}(x)\right]$. We let $\lambda_{i j}^{s}$ denote 0 if the marker $\Lambda_{i j}$ is not attached to any number at the conclusion of stage $s$; otherwise, we take $\lambda_{i j}^{s}=$ the position of $\Lambda_{i j}$ (i.e., the number to which $\Lambda_{i j}$ is attached) at the end of Stage $s$. (It will be clear from the construction that no approximation marker ever occupies more than one position at a time, so that $\lambda_{i j}^{s}$ is well-defined; moreover, it will be evident that for each fixed pair $\left\langle i_{0}, j_{0}\right\rangle$ of numbers the value of $\lambda_{i_{0} j_{0}}^{s}$ changes at most twice as $s \rightarrow \infty$ and that $(\forall s)(\forall i)(\forall j)\left[\lambda_{i j}^{s}>0\right.$ $\left.\Rightarrow(\exists u)(\exists w)\left[\lambda_{i j}^{s}=k(u, w)\right]\right]$.) Finally, we let $p_{0}^{s}$ denote the partial number-theoretic function whose graph is that portion $T_{0}{ }^{s}$ of $T_{0}$ which has been defined by the end of Stage $s$. (When we have finished describing the construction, it will be obvious that $T_{0}{ }^{s}$ is a finite set whose exact contents can be determined effectively from $s$.) 
Stage 0. Attach $\Lambda_{00}$ to $k(0,0)$, set $T_{0}{ }^{0}=\{\langle\hat{k}(0,0), \hat{k}(0,0)\rangle\}$, and go to Stage 1 .

Stage $s+1$. We shall divide our procedure into a series of three steps.

Step A. First, let us suppose that $(\exists e)(\exists k)(\exists l)[e \leqq s+1 \& k \leqq s$ $+1 \& \ell \leqq s+1 \& 0<\lambda_{e k}^{s} \& 0<\lambda_{e+1, \ell}^{s} \&\left\langle\lambda_{e+1, \ell}^{s}, \lambda_{e k}^{s}\right\rangle \in T_{0}^{s} \& g_{0}\left(\lambda_{e k}^{s}, s\right) \geqq$ $\left.\lambda_{e+1, \ell}^{s}\right]$. In this event, let $\left\langle\Lambda_{e 0 k 0}, \Lambda_{e_{0}+1, \ell_{0}}\right\rangle$ be the lexicographically least marker-pair $\left\langle\Lambda_{e k}, \Lambda_{e+1, \ell}\right\rangle$, in terms of the $\pi_{2}$-ordering of $\left\langle\Lambda_{i j}\right\rangle_{i=0, j=0}^{\infty}$, such that $e, k$, and $\ell$ satisfy the foregoing condition. (Note that least here means of highest priority. When our description of the construction is complete, it will be clear that $\lambda_{i j}^{s}$ is a recursive function of $s, i$, and $j$, and that there exists a recursive function $\zeta$ such that $(\forall s)[\zeta(s)$ $\left.=\max \left\{\pi_{2}(i, j) \mid \lambda_{i j}^{s}>0\right\}\right]$. Hence the bound $s+1$ on the quantifiers in the statement of the condition can, if we so desire, be dispensed with.) We attack $\Lambda_{e_{0} k_{0}}$, as follows. Give a barrier marker $\mathcal{B}$ to $t_{0}$, where $t_{0}=$ $(\mu t)\left[(\exists u)\left(\lambda_{e_{0}+1, \ell_{0}}^{s}=\mathcal{k}(t, u)\right)\right]=$ the unique $t$ such that $(\exists u)\left[\lambda_{e_{0}+1, \ell_{0}}^{s}=\right.$ $k(t, u)]$; and give a $\mathcal{B}$ also to each number $m$ such that either $(\exists q)(\exists u)$ $\left[k\left(t_{0}, \mathrm{u}\right) \in \widehat{p_{0}}(k(m, q))\right]$ or $(\exists q)(\exists u)(\exists w)(\exists v)$ [ $m$ bears the reservation $\left.\operatorname{tag} \sum_{w v} \& \hat{k}\left(t_{0}, u\right) \in \widehat{p_{0}}(\boldsymbol{k}(w, q))\right]$. (Note that the last of these two alternatives includes the case: $(\exists v)\left[m\right.$ bears the reservation tag $\left.\Sigma_{t_{0} v}\right]$.) Now, if $z$ is any one of the numbers which have just been given barrier markers and if $r$ is a member of $\rho k_{z}$ such that $(\exists i)(\exists j)\left[r=\lambda_{i j}^{s}\right]$, then we remove from $r$ whichever approximation marker $\Lambda_{i j}$ has been found to be attached to it. Next, take $h_{0}$ to be the smallest number $h$ such that $(\forall \ell)[(\ell$ currently bears a reservation tag or a barrier marker, or some element of $\rho k_{\ell}$ currently bears or has formerly borne an approximation marker) $\Rightarrow h>l]$. (When our description of the construction is complete, it will be evident that $h_{0}$ can be effectively computed from s.) Give $h_{0}$ the reservation tag $\Sigma_{\lambda_{e_{0} k_{0}}^{s} w_{0}}$, where $w_{0}=(\mu w)$ [the tag $\Sigma \lambda_{e_{0} k_{0}}^{s} w_{0}$ has not previously been used in the construction]. (When our description is complete, it will be clear that $\left(n \in \rho p_{0}{ }^{s} \Rightarrow\right.$ $\left.n \in \delta p_{0}{ }^{s}\right) \&\left(n \in \delta p_{0}{ }^{s} \Rightarrow(\exists t) \quad(\exists i) \quad(\exists j)\left[t \leqq s \& n=\lambda_{i j}^{t}\right]\right)$; hence, we see that no member of $\rho \tilde{k}_{h_{0}}$ can be a member of $\delta p_{0}{ }^{s} \cup \rho p_{0}{ }^{s} \cup$ $\left\{x \mid(\exists t)(\exists i)(\exists j)\left[t \leqq s \& i \in N \& j \in N \& x=\lambda_{i j}^{t}\right]\right\}$.) Now proceed to Step B.

If, on the other hand, no such triple $\langle e, k, l\rangle$ exists, go directly to Step B without altering the situations of any markers or tags.

Step B. If there is no number $j$ such that $(\exists y)\left[\langle j, y\rangle \in T_{0}{ }^{s}\right] \&$ $\neg(\exists z)\left[j \neq z \&\langle z, j\rangle \in T_{0}^{s}\right] \& f_{1} *(j)$ does not currently bear a $\mathcal{B}$ and no number currently bears a reservation tag of the form $\Sigma_{j v}$, 
we proceed directly to Step C. Otherwise, let $j_{0}$ be the smallest $j$ such that $(\exists y) \quad\left[\langle j, y\rangle \in T_{0}^{s}\right] \& \neg(\exists z) \quad\left[j \neq z \&\langle z, j\rangle \in T_{0}^{s}\right] \& f_{1}^{*}(j)$ does not currently bear a $\mathcal{B}$ and no number currently bears a reservation tag of the form $\Sigma_{j v}$. (When the construction has been fully described, it will be clear that neither $\mathcal{B}$ 's nor reservation tags are ever removed; hence, it will be seen that the word "currently" is not essential in the specification of $j_{0}$.) Let $m_{0}$ be the smallest number $m$ such that $(\forall n)[(n$ currently bears a reservation tag or a barrier marker, or some element of $\rho k_{n}$ currently bears or has formerly borne an approximation marker) $\Rightarrow m>n$ ]. (Thus, we here specify $m_{0}$ exactly as we $\operatorname{did} h_{0}$ in Step A.) Give $m_{0}$ the reservation tag $\Sigma_{j_{0} v_{0}}$ where $v_{0}=$ $(\mu v)\left[\Sigma_{j_{0}}\right.$ has not previously been used in the construction]; then proceed to Step C.

Step C. We consider $\boldsymbol{\rho} \hat{k}_{(s+1)_{0}}$. If $(s+1)_{0}$ currently bears a $\mathcal{B}$ or if $(s+1)_{0}$ does not currently bear any reservation tag, then, provided $(s+1)_{0}>0$, we set $T_{0}{ }^{s+1}=T_{0}{ }^{s}$ and proceed to Stage $s+2$. If $(s+1)_{0}$ $=0 \& \neg(\exists w)\left[w \leqq s+1 \& \mathcal{k}(0, w) \notin \delta p_{0}{ }^{s}\right]$, then again we set $T_{0}{ }^{s+1}=T_{0}{ }^{s}$ and go on to Stage $s+2$. If $(s+1)_{0}=0 \&(\exists w)[w \leqq$ $\left.s+1 \& \hat{k}(0, w) \notin \delta p_{0} s\right]$, we let $w_{1}=(\mu w)[w \leqq s+1 \& \mathcal{k}(0, w) \notin$ $\left.\delta p_{0}{ }^{s}\right]$; then we define $T_{0}{ }^{s+1}=T_{0}{ }^{s} \cup\left\{\left\langle k\left(0, w_{1}\right), \mathcal{k}\left(0, w_{1}\right)\right\rangle\right\}$, attach $\Lambda_{0 w_{1}}$ to $k\left(0, w_{1}\right)$, and proceed to Stage $s+2$. Finally, suppose that $(s+1)_{0}>0$ and $(s+1)_{0}$ does not currently bear a $\mathcal{B}$, but $(s+1)_{0}$ does currently bear some reservation tag. Then, as will be clear when our account of the construction is complete, there exists just one number $y$, say $y_{0}$, such that $(\exists u)\left[(s+1)_{0}\right.$ bears the tag $\left.\Sigma_{y u}\right]$. If there is no number $n \leqq s+1$ such that $\hat{k}\left((s+1)_{0}, n\right) \notin \delta p_{0}^{s} \& y_{0} \in \hat{f}_{1}(k)(s+$ $\left.1)_{0}, n\right)$ ), we set $T_{0}{ }^{s+1}=T_{0}{ }^{s}$ and go on to Stage $s+2$. Otherwise, let $n_{0}=(\mu n)\left[n \leqq s+1 \& \mathcal{k}\left((s+1)_{0}, n\right) \notin \delta p_{0} s \& y_{0} \in \hat{f}_{1}\left(k\left((s+1)_{0}, n\right)\right)\right]$. Let $z_{0}$ be the uniquely determined number $z$ such that $(\exists v)\left[y_{0}=\right.$ $\lambda_{z v}^{s}$ ]; when we have concluded our description of the construction, it will be plain that there exists exactly one such $z$ corresponding to $y_{0}$. (The existence of at least one such $z$ is assured, since otherwise either $(s+1)_{0}$ would currently bear a $\mathcal{B}$ in virtue of an application of Step $A$ or else no reservation tag of the form $\Sigma_{y_{0} u}$ would as yet have been given to $(s+1)_{0}$; uniqueness is simply a matter of no two approximation markers ever occupying the same position.) Attach $\Lambda_{z_{0}+1, q_{0}}$ to $\hat{k}\left((s+1)_{0}, n_{0}\right)$, where $q_{0}=(\mu q)\left[\Lambda_{z_{0}+1, q}\right.$ has not previously been used in the construction]. Set $T_{0}{ }^{s+1}=T_{0}{ }^{s} \cup\left\{\left\langle\hat{k}\left((s+1)_{0}, n_{0}\right), y_{0}\right\rangle\right\}$ and go to Stage $s+2$.

That completes our description of the construction of the sequence $\left\langle T_{0}{ }^{s}\right\rangle_{s=0}^{\infty}$; the finishing touch is to set $T_{0}=\cup_{s} T_{0} s$. It is obvious from 
the construction that $T_{0}$, so defined, is the graph of a partial recursive function $p_{0}$ such that $\delta p_{0} \subseteq \delta f_{1}$; moreover, it is clear that $(\forall x)$ $\left[x \in \delta p_{0} \Rightarrow p_{0}(x) \in \hat{f}_{1}(x)\right] \& \rho p_{0} \subseteq \delta p_{0}$. Thus $T_{0}$ is a semicomputable tree, since $T_{1}$ is. Furthermore, from what has just been said we see that $T_{0}$ satisfies condition (b) for being a $T_{1}$-skeleton. Next, we observe that $\lim _{s \rightarrow \infty} \lambda_{i j}^{s}$ exists for all pairs $\langle i, j\rangle$. Indeed, since an approximation marker other than $\Lambda_{00}$ can be attached only during Step C of Stage $s+1$ (for some $s$ ) and then only if it has not previously been used in the construction, and since (obviously) $\Lambda_{00}$ is never detached after Stage 0, we readily see that the set of all markers $\Lambda_{i j}$ separates into three mutually exclusive subsets: $M_{0}={ }_{d f}\left\{\Lambda_{i j} \mid \Lambda_{i j}\right.$ is never attached during the construction $\} ; M_{1}={ }_{d f}\left\{\Lambda_{i j} \mid \Lambda_{i j}=\Lambda_{00}\right.$ or $\Lambda_{i j}$ becomes permanently attached during Step $C$ of some stage $s+1\}$; and, finally, $M_{2}={ }_{d f}\left\{\Lambda_{i j} \mid \Lambda_{i j}\right.$ is (necessarily permanently) removed during Step $\mathrm{A}$ of Stage $s+1$, for some $s\}$. Thus, for any fixed pair $\left\langle i_{0}, j_{0}\right\rangle \neq\langle 0,0\rangle$, the total number of values which $\lambda_{i_{0} j_{0}}^{s}$ assumes as $s \rightarrow \infty$ is one if $\Lambda_{i_{0} j_{0}} \in M_{0}$ and two if $\Lambda_{i_{0} j_{0}} \in M_{1} \cup M_{2}$; while the total number of changes of value undergone by $\lambda_{i_{0} j_{0}}$ as $s \rightarrow \infty$ is zero if $\Lambda_{i_{0} j_{0}} \in M_{0}$, one if $\Lambda_{i_{0} j_{0}} \in M_{1}$, and two if $\Lambda_{i_{0} j_{0}} \stackrel{\lambda_{0} j_{0}}{\in} M_{2}$. Hence certainly $\lim _{s \rightarrow \infty} \lambda_{i j}^{s}$ exists for all pairs $\langle i, j\rangle$. We shall henceforth denote $\lim _{s \rightarrow \infty} \lambda_{i j}^{s}$ by $\lambda_{i j}$. Now, it is clear from the manner in which the barrier marker $\mathcal{B}$ is used to "kill" potentially infinite branches of $T_{0}$ that every branch of $T_{0}$ is either finite or of the form $\left\{\lambda_{n y_{n}} \mid n \in N\right\}$ for some sequence $y_{0}, y_{1}, y_{2}, \cdots$. Hence, if $x \in \delta p_{0}$ $\& x \neq p_{0}(x) \& x$ belongs to at least one infinite branch of $T_{0}$ then there must be numbers $k_{0}, j_{0}$, and $\ell_{0}$ such that $x=\lambda_{k_{0}+1, j_{0}} \& p_{0}(x)$ $=\lambda_{k_{0} \ell_{0}}$. Now observe that, since $(\forall n)(\forall m)\left[n \neq m \Rightarrow \rho k_{n} \cap \rho \hat{k}_{m}\right.$ $=\phi] \&(\forall x) \quad\left[\lim _{s \rightarrow \infty} g_{0}(x, s)\right.$ exists], a given marker $\Lambda_{e k}$ can be attacked only finitely many times. Therefore $x>g\left(p_{0}(x)\right)$, since otherwise Step A would eventually force $\Lambda_{k_{0}+1, j_{0}}$ to be permanently removed from $x$. Thus $T_{0}$ is $g$-dominant. Somewhat more tedious is the proof that if $f_{1}$ regresses an infinite set $\alpha$ then $p_{0}$ regresses $\beta$ for some infinite set $\beta \subseteq \alpha$. Suppose that $a_{0}, a_{1}, a_{2}, \cdots$ is a sequence of numbers for which $f_{1}\left(a_{0}\right)=a_{0} \&(\forall n) \quad\left[f_{1}\left(a_{n+1}\right)=a_{n}\right]$; i.e., suppose that $\left\{a_{i} \mid i \in N\right\}$ is an infinite branch of $T_{1}$. Then, in view of Stage 0, Step C of Stage $s+1$, and the fact that $(s+1)_{0}=0$ for infinitely many numbers $s$, we have $(\exists s)(\exists y)\left[a_{0}=\lambda_{0 y}^{s}=\lambda_{0 y} \&\left\langle a_{0}, a_{0}\right\rangle \in T_{0}^{s}\right]$. Let $s_{0}$ be the smallest such $s$, and $y_{0}$ the (uniquely determined) corresponding $y$. Now assume, for the sake of an induction, that there exist a number $s_{m}$, a finite sequence $y_{0}, \cdots, y_{m}$ (reducing to a one-term sequence $y_{0}$ in case $m=0$ ), and a corresponding subsequence $a_{i_{0}}, \cdots, a_{i_{m}}$ of $\left\{a_{i} \mid i \in N\right\}$ such that $a_{i_{0}}=a_{0} \&(\forall j)\left[0 \leqq j \leqq m \Rightarrow a_{i_{j}} \stackrel{i_{0}}{=} \lambda_{j y_{j}}^{s_{m}}=\lambda_{j y_{j}}\right]$ 
$\&\left\langle a_{0}, a_{0}\right\rangle \in T_{0}^{s_{m}} \&(\forall j) \quad\left[0<j \leqq m \Rightarrow\left\langle a_{i_{j}}, a_{i_{j-1}}\right\rangle \in T_{0}^{s_{m}}\right]$. Then, in view of Steps $\mathrm{B}$ and $\mathrm{C}$, there must exist some number $\bar{s}_{1}>s_{m}$ such that at the conclusion of Stage $\bar{s}_{1}$ we have $(\exists k)\left[\Lambda_{m+1, k}\right.$ is attached to some number $w$ for which $\left.a_{i_{m}} \in \hat{f}_{1}(w) \& p_{0} \bar{s}_{1}(w)=a_{i_{m}}\right]$. Now, it may happen that, on account of Step $A, f_{1}^{*}(w)$ receives a $\mathcal{B}$ at some stage $\bar{s}_{2}$ in the construction; however, if this occurs then some number $h>f_{1}{ }^{*}(w)$ receives a reservation tag $\Sigma_{a_{i_{m} \ell} \text {, for some }}$ $\ell$, at Stage $\bar{s}_{2}$. Clearly, we have $\bar{s}_{2} \geqq \bar{s}_{1}$ (since attaching a $\mathcal{B}$ to $x$ forces removal of all approximation markers which happen to be attached to members of $\rho k_{x}$, and since no barrier marker is ever $r e$ moved); so, there will be a stage $\bar{s}_{3}, \bar{s}_{3} \geqq \bar{s}_{2} \geqq \bar{s}_{1}$, at which Step C compels us to attach a marker of the form $\Lambda_{m+1, y}$ to some number $z \in \rho \hat{k}_{h}$ and place the pair $\left\langle z, a_{i_{m}}\right\rangle$ in $T_{0}{ }^{\bar{s}_{3}} . \rho \hat{k}_{h}$ may later cease, in virtue of the exigencies of Step $\mathrm{A}$, to be a source of new $p_{0}$-preimages of $a_{i_{m}}$; if so, however, then it is subsequently replaced (in the manner just indicated for $\left.\rho k_{f_{1}^{*}(w)}\right)$ by some new source, $\rho k_{r}$, of such preimages. This process of "changing our minds about the set of desirable $p_{0^{-}}$ preimages of $a_{i_{m}}$ " can be iterated only finitely often, since (i) $\lim _{s \rightarrow \infty} g_{0}\left(a_{i_{m}}, s\right)$ exists and (ii) the sets $\rho k_{x}, x \in N$, are mutually disjoint. Hence, there must be a stage $s^{\prime}>s_{m}$ such that $(\exists j)\left[\lambda_{m+1, j}^{s^{\prime}}\right.$ $\left.=\lambda_{m+1, j} \in \delta f_{1} \& a_{i_{m}}=p_{0}{ }^{s^{\prime}}\left(\lambda_{m+1, j}^{s^{\prime}}\right)\right]$. It is easily seen by inspection of the construction that there is in fact a uniquely determined $j$, say $j_{1}$, such that $\lambda_{m+1, j}^{s^{\prime}}=\lambda_{m+1, j} \in \delta f_{1} \& a_{i_{m}}=p_{0}{ }^{s^{\prime}}\left(\lambda_{m+1, j}^{s^{\prime}}\right)$. Moreover, there is a uniquely determined number $\ell$, say $\ell_{1}$, such that $\lambda_{m+1, j_{1}} \in \rho \hat{k}_{\ell}$. Now, since $\left\{a_{i} \mid i \in N\right\}$ is an infinite branch of $T_{1}$, there exists a uniquely determined index $t$, say $t_{1}$, such that $a_{t} \in \rho k_{\ell_{1}}$. Since $\Lambda_{m+1, j_{1}}$ is permanently attached to a member of $\rho k_{\ell_{1}}, \ell_{1}$ cannot ever bear a $\mathcal{B}$; hence, in view of Step $C$, we have $\left\langle a_{t_{1}}, a_{i_{m}}\right\rangle \in T^{s_{m+1}}$ for some number $s_{m+1} \geqq s^{\prime}$. So, if we set $i_{m+1}=t_{1}$ then the conjunction $a_{i_{0}}=a_{0} \&(\forall j) \quad\left[0 \leqq j \leqq m+1 \Rightarrow a_{i_{j}}=\lambda_{j y_{j}}^{s m+1}=\lambda_{j y_{j}}\right] \&\left\langle a_{0}, a_{0}\right\rangle$ $\in T_{0}^{s_{m+1}} \&(\forall j)\left[0<j \leqq m+1 \Rightarrow\left\langle a_{i_{j}}, a_{i_{j-1}}\right\rangle \in T_{0}^{s_{m+1}}\right]$ holds for a suitably specified sequence $y_{0}, y_{1}, \cdots, y_{m+1}$. It follows by induction that some infinite subset of $\left\{a_{i} \mid i \in N\right\}$ is a branch of $T_{0}$. It now remains only to be shown that $T_{0}$ is finite-branching if $T_{1}$ is finitebranching and that $T_{0}$ is uniformly co-enumerable relative to $T_{1}$, i.e., that there is a fixed r.e. set $\boldsymbol{\gamma}$ such that $(\forall \boldsymbol{\alpha})(\forall \boldsymbol{\beta})[(\boldsymbol{\alpha}$ an infinite branch of $T_{1} \& \beta$ an infinite branch of $\left.T_{0} \& \beta \subseteq \alpha \Rightarrow \alpha-\beta=\alpha \cap \gamma\right]$ (for if such a $\gamma$ exists then, obviously, it is also the case that each infinite branch $\alpha$ of $T_{1}$ can include at most one infinite branch $\beta$ of $T_{0}$ ). But to verify the uniform co-enumerability condition, we need only note that (because of the "largeness criterion" according to which $h_{0}$ is determined in Step A) $p_{0}$ satisfies the condition: $(\forall m)(\forall n)(\forall q)$ 
$\left[\left(m \in \delta p_{0} \& n=p_{0}(m) \& q \in \hat{f}_{1}(m)-\{m\} \& n \in \hat{f}_{1}(q)-\{q\}\right)\right.$ $\Rightarrow\left(q \in \delta p_{0} \Rightarrow f_{1} *(q)\right.$ eventually bears a $\mathcal{B}$ and hence no member of $\rho \widehat{K}_{f_{1}^{*}(q)}$ belongs to an infinite branch of $\left.\left.T_{0}\right)\right]$. For, from this special feature of $p_{0}$ it follows easily that if $\gamma=\left\{n \mid n \in \delta f_{1} \&(\exists w)(\exists z)\right.$ $\left.\left[n \in \hat{f}_{1}(w)-\{w\} \& z \in \hat{f_{1}}(n)-\{n\} \& p_{0}(w)=z\right]\right\}$ then $\gamma$ is a recursively enumerable set such that ( $\alpha$ an infinite branch of $T_{1} \& \beta$ an infinite branch of $\left.T_{0} \& \beta \subseteq \alpha\right) \Rightarrow \alpha-\beta=\alpha \cap \gamma$. Finally, $T_{0}$ is finitebranching if $T_{1}$ is. For, given $x_{0} \in \rho p_{0}$, it is clear from the construction that if $x_{0}$ is drawing new $p_{0}$-preimages from $\rho k_{w_{0}}$ at stage $s$ then it continues to draw them solely from $\rho k_{w_{0}}$ at all stages $t>s$ unless $g_{0}\left(x_{0}, t\right) \neq g_{0}\left(x_{0}, t-1\right)$ for some $t>s$. Thus, all the $p_{0}$-preimages of $x_{0}$ lie in the union of just finitely many sets $\rho \hat{k}_{w}$. The proof of Theroem 2.2 is therewith complete.

2.3. Remarks. (1): Our technique in proving Theorem 2.2 derives from Yates' proof of [20, Theorem 3]. In the latter proof, Yates showed that a cohesive $\Pi_{1}^{0}$ set can be so constructed as to bound the principal function of a given (infinite) $\Sigma_{2}^{0}$ set. We can analogously strengthen Theorem 2.2, replacing the arbitrary $\Delta_{2}$ function in the statement of the theorem by the principal function of an arbitrarily given infinite $\Sigma_{2}^{0}$ set. All that is required for such a strengthening is to observe that every infinite $\Sigma_{2}^{0}$ set has an infinite subset recursive in $0^{1}$ and that such a subset can be enumerated in increasing order by a $\Delta_{2}$ function. (2): After seeing a pre-print of this article, Alfred Manaster devised a proof of Theorem 2.2 which is similar to but shorter than our above proof. We are inclined to prefer the proof as given above, however, since it seems to us that it affords a slightly more dynamic view of the construction of $T_{0}$ from $T_{1}$ than does Manaster's more concise version.

We further observe at this point that A. N. Degtev has given an independent construction for a particular co-r.e. $t$-retraceable set, in terms of the "deficiency sets" of recursive functions (cf. [19]). (For the definition of " $t$-retraceable" see $\$ 3$ below.) His procedure can be adapted, without very much difficulty, to the problem of proving Theorem 2.2 and its corollaries in the special case of regressive sets with r.e. complements; for regressive sets in general, however, there would seem to be no routine extension of his technique which would lead to the full Theorem 2.2. Thus, from a general point of view, it would appear better to work with trees in a direct manner, as in the present article.

Let $\left.T_{0}\right\}_{*} T_{1}$ mean that $T_{0}-3 T_{1} \&(\forall \boldsymbol{\alpha})(\forall \boldsymbol{\beta})$ [ $(\boldsymbol{\alpha}$ an infinite branch of $T_{1} \& \beta$ an infinite branch of $\left.T_{0} \& \beta \subseteq \alpha\right) \Rightarrow \alpha-\beta$ is recursively enumerable in $\alpha$ ]. (Recall that a set $\tau_{1}$ is said to be recursively enumer- 
able in a set $\tau_{2}$ just in case $\tau_{1}=\rho f$ where $f$ is a function recursive in $\tau_{2}$.) The ease with which we were able to build uniform coenumerability into our construction of $T_{0}$ in the proof of Theorem 2.2 might cause the reader to wonder just how much is added to $T_{1^{-}}$ skeletonhood by requiring specifically that $T_{0}$ be a uniformly coenumerable $T_{1}$-skeleton. Before proceeding to $\$ 3$, we pause to prove a theorem which shows that there is, in fact, a considerable gap even between the relations $T_{0}-3_{*} T_{1}$ and $T_{0}-3_{+} T_{1}$.

2.4. Theorem. Let $\beta$ be an arithmetical subset of $N$, and let $\left\{W_{n}{ }^{\beta}\right\}_{n=0}^{\infty}$ be an enumeration of all sets recursively enumerable in $\beta$. Then there exist semicomputable trees $T_{0}$ and $T_{1}$ such that

(2.4i) $T_{1}$ has a unique infinite branch;

(2.4ii) $T_{0}-{ }_{*} T_{1}$; and

(2.4iii) $\left(\forall \alpha_{0}\right)\left(\forall \alpha_{1}\right)\left[\left(\alpha_{0}=\right.\right.$ an infinite branch of $T_{0} \& \alpha_{1}=$ an infinite branch of $\left.T_{1} \& \alpha_{0} \subseteq \alpha_{1}\right) \Rightarrow(\forall m) \quad\left[\alpha_{1}-\alpha_{0} \neq \alpha_{1} \cap\right.$ $\left.W_{m}^{\beta}\right]$ ].

Proof. Since $\beta$ is arithmetical, there exists a number $n_{0}$ such that $\boldsymbol{\beta} \leqq \mathbf{0}^{\left(n_{0}\right)}$. (Following the (standard) practice of [13], we denote by $\alpha$ the degree (of unsolvability) of a given set $\alpha \subseteq N$ and by $0^{(m)}$ the degree of the $m$-th jump of the empty set; $\leqq$ denotes lessthan-or-equal-to in the sense of the upper semilattice of degrees of unsolvability.) Now, for any $n \in N$ there exist a $\Pi_{n+1}^{0}$ set $\alpha_{1}$ and a $\Pi_{n+2}^{0}$ set $\gamma$ such that $\alpha_{1}$ is the unique infinite branch of a semicomputable tree $T_{1}$ satisfying $(\forall x)(\forall y)\left[\langle x, y\rangle \in T_{1} \Rightarrow x \geqq y\right], \gamma$ is the unique infinite branch of a semicomputable tree $T$ satisfying $(\forall x)(\forall y)$ $[\langle x, y\rangle \in T \Rightarrow x \geqq y], \quad \alpha_{1}=0^{(n+1)}, \quad$ and $\quad \gamma=0^{(n+2)} ; \quad$ in case $n \geqq 1$ this follows from [13, Theorem 4.14 (2)], while for $n=0$ we appeal also to [7, Theorem T3] and [19, Theorem 2]. Let $\alpha_{1}, \gamma$ be so chosen, with $n=n_{0}$. Let $p, q$ be the partial recursive functions defining $T_{1}, T$, respectively. Following the procedure used in the proof of [7, Proposition P4], we define a partial recursive function $\omega$ by the condition that $\langle x, y\rangle \in$ the graph of $\omega \Leftrightarrow\left[x \in \delta p \& p^{*}(x) \in\right.$ $\left.\delta q \& y \in \hat{p}(x) \& p^{*}(y)=q\left(p^{*}(x)\right)\right]$. It is easily checked that $\omega$ defines a tree $T_{0}$ whose unique infinite branch $\alpha_{0}$ is the range of the composite function $p_{\alpha_{1}}\left(p_{\gamma}(x)\right)$, where $p_{\alpha_{1}}$ enumerates $\alpha_{1}$ in natural order and $p_{\gamma}$ enumerates $\gamma$ in natural order. Clearly $\alpha_{0} \subseteq \alpha_{1}$, and so (noting that $\delta \omega \subseteq \delta p \&(\forall x) \quad[x \in \delta \omega \Rightarrow \omega(x) \in \hat{p}(x)])$ we have $T_{0}-3 T_{1}$. Moreover, since $(\forall x)\left[x \in \alpha_{0} \Leftrightarrow\left(x \in \alpha_{1} \& p^{*}(x) \in \gamma\right)\right]$ we have that $\alpha_{0}$ is a $\Pi_{n_{0}+2}^{0}$ set. Thus $N-\alpha_{0}$ is $\Sigma_{n_{0}+2}^{0}$, so that $N-\alpha_{0}$ is recursively enumerable in any set of degree $0^{\left(n_{0}+1\right)}$. In particular, then, $N-\alpha_{0}$ 
is recursively enumerable in $\alpha_{1}$. It follows that $\left(N-\alpha_{0}\right) \cap \alpha_{1}$, i.e., $\alpha_{1}-\alpha_{0}$, is recursively enumerable in $\alpha_{1}$; hence $T_{0}-3_{*} T_{1}$. Finally, if $(\exists m)\left[\alpha_{1}-\alpha_{0}=\alpha_{1} \cap W_{m}{ }^{\beta}\right]$ then (since $(\forall m)\left\{W_{m}{ }^{\beta}\right.$ is recursive in $\left.\left.0^{\left(n_{0}+1\right)}\right]\right)$ we have $\boldsymbol{\alpha}_{0} \leqq \mathbf{0}^{\left(n_{0}+1\right)}$. But it is easy to see that $\boldsymbol{\alpha}_{0} \leqq 0^{\left(n_{0}+1\right)} \Rightarrow \boldsymbol{\gamma} \leqq \boldsymbol{\alpha}_{1}$. Hence, since $\boldsymbol{\gamma}$ is strictly larger than $\alpha_{1}$, we conclude that $(\forall m)\left[\alpha_{1}-\alpha_{0} \neq \alpha_{1} \cap W_{m}^{\beta}\right]$, and the proof is complete.

2.5. Remark. Theorem 2.4 is by no means a comprehensive survey of the discrepancy between $-3_{*}$ and $-_{+}$for semicomputable trees; in particular, one might inquire about a precise violation of uniformity, in the sense of the existence of semicomputable trees $T_{0}$ and $T_{1}$ such that $T_{0}-3 T_{1} \&\left(\forall \alpha_{0}\right)\left(\forall \alpha_{1}\right)\left[\left(\alpha_{0}=\right.\right.$ an infinite branch of $T_{0} \& \alpha_{1}=$ an infinite branch of $\left.\left.T_{1} \& \alpha_{0} \subseteq \alpha_{1}\right) \Rightarrow(\exists n)\left[\alpha_{1}-\alpha_{0}=\alpha_{1} \cap W_{n}\right]\right] \&$ $\neg(\exists n)\left(\forall \alpha_{0}\right)\left(\forall \alpha_{1}\right)\left[\left(\alpha_{0}=\right.\right.$ an infinite branch of $T_{0} \& \alpha_{1}=$ an infinite branch of $\left.\left.T_{1} \& \alpha_{0} \subseteq \alpha_{1}\right) \Rightarrow \alpha_{1}-\alpha_{0}=\alpha_{1} \cap W_{n}\right]$. We think that such pairs of trees exist, but do not at present have any clear ideas about proving it. Also left open are the questions whether Theorem 2.4 can be extended into the hyperarithmetical hierarchy, and whether an example can be found in which $(2.4 i)$ is replaced by the condition that $T_{1}$ have uncountably many branches. Finally, we mention that the proof of Theorem 4.14 (2) of [13] is sufficiently effective to allow us to prove the following, where $-3_{+}{ }^{A}$ is defined exactly as is $-_{+}$except that $\gamma$ is allowed to be any fixed arithmetical subset of $N$ : There are semicomputable trees $T_{1}$ and $T_{0}$ such that (i) $T_{1}$ has $\boldsymbol{\kappa}_{0}$ infinite branches, each of which is arithmetical, (ii) $T_{0}-3_{*} T_{1}$, and (iii) $T_{\mathrm{o}}+3_{+}{ }^{A} T_{1}$.

3. Some applications to the class of regressive isols. In this section, we shall indicate a few applications of Theorem 2.2 within its most obvious domain of relevance: the theory of regressive isols. (For an excellent survey of the basic results on regressive isols, the reader is referred to Dekker's paper [10]; more recent results can be found, for example, in [3], [4], [5], [6], [12], and [17]. Some of these articles, as well as some others less recent (e.g. [2]), contain results which can be immediately sharpened by applying the "thinning" technique of the present paper.)

Following well-established notational practice, we let $\boldsymbol{\Lambda}_{\boldsymbol{R}}\left(\boldsymbol{\Lambda}_{\mathrm{ZR}}\right)$ denote the class of regressive (co-simple regressive) isols ([10]); and we let $\Lambda^{*}$ denote the ring of isolic integers, defined in [8, Chapter XI]. $\boldsymbol{\Lambda}_{R}^{*}\left(\boldsymbol{\Lambda}_{\mathrm{Z} R}^{*}\right)$ will denote the subring of $\boldsymbol{\Lambda}^{*}$ generated by $\boldsymbol{\Lambda}_{R}$ (by $\Lambda_{\mathrm{ZR}}$ ). Since it is customary to include the finite isols (i.e., the natural numbers) among the members of both $\Lambda_{R}$ and $\Lambda_{\mathrm{ZR}}$, we shall use $\boldsymbol{\Lambda}_{\boldsymbol{R}}{ }^{\infty}$ (respectively $\boldsymbol{\Lambda}_{\mathrm{Z} R}^{\infty}$ ) to denote the class of infinite re- 
gressive (infinite co-simple regressive) isols; and similarly for the notations $\Lambda_{R}^{* \infty}, \Lambda_{Z R}^{* \infty}$.

As shown in [9], each member of $\Lambda_{R}$ has a retraceable representative. We shall say that an isol $\boldsymbol{A}$ is invariantly retraceable provided that $(\forall \boldsymbol{\alpha})[\boldsymbol{\alpha} \in \boldsymbol{A} \Rightarrow \boldsymbol{\alpha}$ is retraceable]. (Our use of boldface capital roman letters for isols will not clash with our boldface notation for degrees, since we never use capital roman letters for degrees.) Let $A$ be called hereditarily invariantly retraceable $\Leftrightarrow_{d f}(\forall \mathrm{B})[\mathrm{B} \leqq$ $\mathbf{A} \Rightarrow \mathbf{B}$ is invariantly retraceable]. (Here, of course, $\leqq$ means isolic less-than-or-equal-to as defined in [8].) It is a simple exercise to show that invariant retraceability implies hereditary invariant retraceability, so that the two concepts are in fact equivalent. By a totally retraceable isol we shall mean an isol A such that for every regressive isol $\mathbf{B}$ and every pair of sets $\alpha, \beta$, we have $(\alpha \in A \&$ $\beta \in \mathbf{B} \& \beta \subseteq \alpha) \Rightarrow \mathbf{B}$ is invariantly retraceable. Let $\exists \subset$ denote the class of all totally retraceable isols. Theorem 1 of [14], which we shall here obtain (in strengthened form) as Theorem 3.4, implies the existence of a rich supply of isols belonging to the class $\square \mathcal{R}$. In preparation for this result, we shall now derive an appropriate specialization of Theorem 2.2 .

3.1 Theorem. Let $T_{1}$ be a non-trivial semicomputable tree, and let $f_{1}$ be the partial recursive function whose graph is $T_{1}$; we assume $0 \notin \rho f_{1}$. (The latter assumption is inessential; it is only a matter of convenience.) Then there exists a semicomputable tree $T_{0}$ such that $T_{0}-3_{+} T_{1}, T_{0}$ is finite-branching if $T_{1}$ is, and $T_{0}$ has in addition the following property, where $p_{0}$ is the partial recursive function whose graph is $T_{0}$ :

$$
(\forall x)\left[x \in \delta p_{0} \Rightarrow p_{0}(x) \leqq x\right] \&
$$

$(\forall \alpha)(\forall d)(\forall e)(\forall n)(\forall m)\left[\left(\alpha \subseteq\right.\right.$ a branch of $T_{0} \& \alpha$ infinite \& $p_{\alpha}=$ the function from $N$ into $N$ which enumerates $\alpha$ in order of magnitude $\& \max \{e, d\} \leqq n<m \& \varphi_{d}^{1}$ is one-to-one on $\delta \varphi_{d}{ }^{1} \&\left\{p_{\alpha}(n), p_{\alpha}(m)\right\} \subseteq$ $\left.\left.\delta \varphi_{d}^{1} \& \varphi_{d}^{1}\left(p_{\alpha}(n)\right) \in \delta \varphi_{e}{ }^{1}\right) \rightarrow \varphi_{e}{ }^{1}\left(\varphi_{d}^{1}\left(p_{\alpha}(n)\right)\right)<\varphi_{d}^{1}\left(p_{\alpha}(m)\right)\right]$.

Proof. By Theorem 2.2, there exists a semicomputable tree $T_{2}$ defined by a partial recursive function $p_{2}$ such that $T_{2}-3_{+} T_{1} \& T_{2}$ is finite-branching if $T_{1}$ is \& $T_{2}$ is $\xi$-dominant where $\xi$ is the identity function: $\xi(x)=x$ for all $x$. Then, as is easily seen, $p_{2}$ retraces each infinite branch of $T_{2}$. There is no loss of generality in assuming, in fact, that $(\forall x)\left[x \in \delta p_{2} \Rightarrow p_{2}(x) \leqq x\right]$; for any such modification of $p_{2}$ as may be needed to actually achieve this can affect only the finite branches of $T_{2}$, and we can easily insure retention of property (b) of 
$\$ 1$. We shall next define a $\Delta_{2}$ function $\Psi: N \rightarrow N$ such that if $T_{0}$ is any $\Psi$-dominant uniformly co-enumerable $T_{2}$-skeleton (which is finite-branching provided $T_{2}$ is) then $T_{0}$ meets the demands of the theorem. The definition of $\Psi$ in terms of $p_{2}$ is as follows:

$$
\Psi(x)=\left\{\begin{array}{l}
0, \quad \text { if } x=0 ; \\
1+\Psi(x-1), \text { if } x>0 \& x \notin \delta p_{2} ; \\
(\mu z)[z>\Psi(x-1) \& \\
(\forall d)(\forall e)(\forall k)(\forall \ell)(\forall j)[(d \leqq x \& e \leqq x \& \\
\varphi_{d}^{1} \text { is one-to-one on } \delta \varphi_{d}^{1} \& k \in \hat{p}_{2}(x) \cap \delta \varphi_{d}^{1} \& \\
\left.\ell=\varphi_{d}^{1}(k) \& \ell \in \delta \varphi_{e}^{1} \& j=\varphi_{e}{ }^{1}(\ell)\right) \Rightarrow \\
\left.(\forall w)\left[\left(w \in \delta \varphi_{d}^{1} \& j \geqq \varphi_{d}^{1}(w)\right) \Rightarrow z>w\right]\right] \& \\
(\forall d)(\forall k)(\forall \ell)\left[\left(d \leqq x \& \varphi_{d}^{1}\right.\right. \text { is } \\
\text { one-to-one on } \delta \varphi_{d}^{1} \& k \in \hat{p}_{2}(x) \cap \delta \varphi_{d}^{1} \& \ell= \\
\left.\left.\varphi_{d}^{1}(k)\right) \Rightarrow(\forall w)\left[\left(w \in \delta \varphi_{d}^{1} \& \ell \geqq \varphi_{d}^{1}(w)\right) \Rightarrow z>w\right]\right] \\
\quad \text { if } x>0 \& x \in \delta p_{2} .
\end{array}\right.
$$

It is easily verified that $\Psi$ is a strictly increasing $\Delta_{2}$ function. Hence, by Theorem 2.2, $T_{2}$ has a uniformly co-enumerable $T_{2}$-skeleton $T_{0}$ such that $T_{0}$ is $\Psi$-dominant and is finite-branching if $T_{2}$ is. Let $p_{0}$ be the partial recursive function whose graph is $T_{0}$. We note that (by the definition of the relation-3) we have $\delta p_{0} \subseteq \delta p_{2} \&$ $(\forall x)\left[x \in \delta p_{0} \Rightarrow p_{0}(x) \in \hat{p}_{2}(x)\right] ;$ moreover, we have $0 \notin \rho p_{2}$, since $\rho p_{2} \subseteq \rho f_{1} \& 0 \notin \rho f_{1}$. These conditions imply that $0 \notin \rho p_{0}$; and they further imply that every infinite branch of $T_{0}$ is retraced by $p_{0}$. Now let $\alpha$ be an infinite subset of some branch $\tau$ of $T_{0}$; and let $p_{\alpha}$ be the function which enumerates $\alpha$ in order of magnitude. Let $d$ be a number such that $\varphi_{d}{ }^{1}$ is one-to-one on $\delta \varphi_{d}{ }^{1}$, and let $e \in N$. Suppose $n \geqq \max \{d, e\}, m>n,\left\{p_{\alpha}(n), p_{\alpha}(m)\right\} \subseteq \delta \varphi_{d}{ }^{1}$, and $\varphi_{d}{ }^{1}\left(p_{\alpha}(n)\right) \in \delta \varphi_{e}{ }^{1}$. Since $\rho p_{0} \subseteq \delta p_{0} \subseteq \delta p_{2} \&(\forall x)\left[x \in \delta p_{0} \Rightarrow p_{0}(x) \in \hat{p}_{2}(x)\right]$, we see that $p_{\alpha}(n) \in \hat{p}_{2}\left(p_{0}\left(p_{\alpha}(m)\right)\right)$; also, $p_{0}\left(p_{\alpha}(m)\right)>0$ since $0 € \rho p_{0}$. Hence, by the definition of $\Psi, \Psi\left(p_{0}\left(p_{\alpha}(m)\right)\right)$ is larger than $w$ for every number $w$ such that $w \in \delta \varphi_{d}^{1} \& \varphi_{d}^{1}(w) \leqq \varphi_{e}{ }^{1}\left(\varphi_{d}^{1}\left(p_{\alpha}(n)\right)\right)$. But, since $T_{0}$ is $\Psi$ dominant, we therefore have that $p_{\alpha}(m)$ is larger than $w$ for every $w$ satisfying $w \in \delta \varphi_{d}^{1} \& \varphi_{d}^{1}(w) \leqq \varphi_{e}^{1}\left(\varphi_{d}^{1}\left(p_{\alpha}(n)\right)\right)$. Thus $\varphi_{d}^{1}\left(p_{\alpha}(m)\right)>$ $\varphi_{e}^{1}\left(\varphi_{d}^{1}\left(p_{\alpha}(n)\right)\right)$, and we have completed the proof. (Note that the condition $\delta p_{0} \subseteq \delta p_{2} \&(\forall x)\left[x \in \delta p_{0} \Rightarrow p_{0}(x) \in \hat{p}_{2}(x)\right]$ guarantees $(\forall x)$ $\left[x \in \delta p_{0} \Rightarrow p_{0}(x) \leqq x\right]$ and so insures the initial clause in $(*)$.) 
Our next theorem singles out for special notice two related, useful properties of (branches of) those trees $T_{0}$ which can be obtained from given trees by applying Theorem 3.1. Let us say, for want of better terminology, that a non-trivial semicomputable tree $T_{0}$ defined by a partial recursive function $p_{0}$ is uniformly thin-branched $\Leftrightarrow_{d f}$ the pair $\left\langle T_{0}, p_{0}\right\rangle$ satisfies the condition $(*)$ in the statement of Theorem 3.1. Property (2) below is one which various other authors (e.g., Ellentuck, J. Gersting, and A. N. Degtev) have studied under a variety of labels (but not in the present conceptual framework of trees). Property (1) is a standard domination property.

3.2. Theorem. Let $T_{0}$ be a uniformly thin branched, non-trivial, semicomputable tree; and let $p_{0}$ be the partial recursive function whose graph is $T_{0}$. If $\tau$ is an infinite branch of $T_{0}$ and $p_{\tau}$ is the function which enumerates $\tau$ in order of magnitude and $\varphi_{e}{ }^{1}$ is any partial recursive function, then

$$
\begin{aligned}
& \left(\exists m_{1}\right)(\forall n)\left[\left(n \geqq m_{1} \& n \in \delta \varphi_{e}{ }^{\mathrm{l}}\right) \Rightarrow p_{\tau}(n) \geqq \varphi_{e}^{\mathrm{l}}(n)\right], \text { and } \\
& \left(\exists m_{2}\right)(\forall n)\left[\left(n \geqq m_{2} \& n \in \tau \& p_{0}(n) \in \delta \varphi_{e}\right)^{1} \Rightarrow n>\varphi_{e}{ }^{\mathrm{l}}\left(p_{0}(n)\right)\right] .
\end{aligned}
$$

Proof. Letting $T_{1}$ be any semicomputable tree such that $T_{0}-_{+} T_{1}$ (e.g., take $T_{1}=T_{0}$ ), take $\varphi_{d}{ }^{1}=$ the identity function and apply Theorem 3.1; since $p_{0}(n)<n$ for all those $n \in \delta p_{0}$ such that $p_{0}{ }^{*}(n)$ $>0$, part (2) follows at once. For part (1), let a partial recursive function $p_{1}$ be defined as follows: $\langle x, y\rangle \in$ the graph of $p_{1} \Leftrightarrow{ }_{d f} x \in \delta p_{0} \&$ $p_{0}{ }^{*}(x)+1 \in \delta \varphi_{e}{ }^{1} \& y=\varphi_{e}{ }^{1}\left(p_{0}{ }^{*}(x)+1\right)$. Let $u$ be a number such that $\varphi_{u}{ }^{1}=p_{1}$. By part (2), there is a number $m_{2}$ such that $\left(n \geqq m_{2} \&\right.$ $\left.n \in \tau \& p_{0}(n) \in \delta \varphi_{u}{ }^{1}\right) \Rightarrow n>\varphi_{u}{ }^{1}\left(p_{0}(n)\right)$. Now, clearly, $p_{0}{ }^{*}\left(p_{\tau}(n)\right)=n$ for all $n$, since $p_{0}$ retraces $\tau$. We set $m_{1}=m_{2}+1$. Suppose $n \geqq m_{1}$ $\& n \in \delta \varphi_{e}{ }^{1}$. Then $p_{0}{ }^{*}\left(p_{\tau}(n-1)\right)=n-1$. Since $(n-1)+1=$ $n \in \delta \varphi_{e}^{1}$, we have that $\varphi_{u}^{1}\left(p_{\tau}(n-1)\right)$ is defined and $=\varphi_{e}{ }^{1}(n)$. But therefore, since $p_{\tau}(n) \geqq n>m_{2} \& p_{\tau}(n) \in \tau \& p_{0}\left(p_{\tau}(n)\right)=p_{\tau}(n-1) \in$ $\delta \varphi_{u}{ }^{1}$, we get $p_{\tau}(n)>\varphi_{u}^{1}\left(p_{0}\left(p_{\tau}(n)\right)\right)=\varphi_{u}^{1}\left(p_{\tau}(n-1)\right)=\varphi_{e}{ }^{1}(n)$. (1) is thus verified, taking $m_{1}=m_{2}+1$ as just indicated.

3.3 ReMark. Our proof of Theorem 3.2 shows that the implication $(2) \Rightarrow(1)$ holds for all retraceable sets. Regarding the converse, it can rather easily be shown that $(1) \Rightarrow(2)$ does not hold for all retraceable sets. In fact, while it can easily be shown that $\rho p_{\tau_{2}} p_{\tau_{1}}$ has property (1) if $\tau_{1}$ does $\left(\tau_{1}, \tau_{2}\right.$ infinite), one can construct infinite co-r.e. retraceable sets $\tau_{1}$ and $\tau_{2}$ such that $\tau_{1}$ has property (2) while $\rho p_{\tau_{2}} p_{\tau_{1}}$ does not.

An infinite set $\tau$ which satisfies condition (2) in the statement of Theorem 3.2 (relative to a partial recursive function $p_{0}$ such that $p_{0}$ 
retraces $\tau$ ) is termed T-retraceable in [11]. (As noted in [11], the terminology originated with Judith Gersting.) To avoid any possible confusion with our notation for trees, we shall here change to lower case and refer to such sets as being $t$-retraceable. By the obvious extension of terminology, an isol $\boldsymbol{A}$ is said to be $t$-retraceable provided it contains at least one t-retraceable set. That each infinite branch $\tau$ of a uniformly thin-branched tree is $t$-retraceable is just part (2) of Theorem 3.2 above; that each such $\tau$ is totally t-retraceable is an immediate consequence of Theorem 3.4 below.

From now on, we shall use $[\alpha]$ as an alternative form of notation for the isol $A$ satisfying $\alpha \in A$; if $\alpha$ is represented as (the range of) a sequence $\left\langle a_{i}\right\rangle_{i=0}^{\infty}$, then $\left[\left\langle a_{i}\right\rangle_{i=0}^{\infty}\right]$ is also admitted as a notation for the isol containing $\alpha$. We shall say that an infinite isol $[\alpha]$ is totally $t$-retraceable $\Leftrightarrow_{d f}(\forall B)(\forall \beta) \quad(\forall \gamma) \quad(\forall \tau) \quad[(\tau \in[\alpha] \& \beta \in B \& \gamma \in$ $B \& B$ is infinite and regressive $\& \beta \subseteq \tau) \Rightarrow \gamma$ is a $t$-retraceable set]. It is obvious that $[\alpha]$ totally $t$-retraceable $\Rightarrow[\alpha]$ totally retraceable. We are now ready to derive our main corollary to Theorem 2.2.

3.4 Theorem. (cf. [14, Theorem 1]). Let $T_{0}$ be any uniformly thin-branched, non-trivial, semicomputable tree. Then $(\forall \tau)[(\tau=$ an infinite branch of $\left.T_{0}\right) \Rightarrow[\tau]$ is totally t-retraceable $]$. Thus every nontrivial semicomputable tree $T_{1}$ admits a semicomputable, uniformly co-enumerable $T_{1}$-skeleton $T_{0}$ such that $T_{0}$ is finite-branching if $T_{1}$ is and each infinite branch of $T_{0}$ determines a totally t-retraceable isol. In particular, if $W_{n}$ is a recursively enumerable set whose complement is infinite, immune, and regressive, then there exists a recursively enumerable set $W_{m}$ such that $W_{n} \subseteq W_{m}$ \& the complement of $W_{m}$ represents an infinite, totally $t$-retraceable isol.

Proof. Suppose $\tau$ is an infinite branch of $T_{0}$; and let $\alpha$ be an infinite subset of $\tau$. Let $\varphi_{d}{ }^{1}$ be a one-to-one partial recursive function such that $\alpha \subseteq \delta \varphi_{d}{ }^{1}$; and suppose $\varphi_{e}{ }^{1}$ is a partial recursive function which regresses $\varphi_{d}{ }^{1}(\alpha)$. By $(*), m>n \geqq \max \{d, e\} \Rightarrow \varphi_{e}{ }^{1}\left(\varphi_{d}{ }^{1}\left(p_{\alpha}(n)\right)\right)$ $<\varphi_{d}^{1}\left(p_{\alpha}(m)\right)$. But also, if $m>n \geqq \max \{e, d\} \geqq d$, (*) yields $\varphi_{d}^{1}\left(p_{\alpha}(n)\right)<\varphi_{d}^{1}\left(p_{\alpha}(m)\right)$. (Simply take $e=0$ so that $\varphi_{e}{ }^{1}=$ the identity function.) Thus, there exists a fixed finite set $F$ such that $(n \geqq$ $\left.\max \{e, d\} \& \varphi_{e}{ }^{1}\left(\varphi_{d}^{1}\left(p_{\alpha}(n)\right)\right)>\varphi_{d}^{1}\left(p_{\alpha}(n)\right)\right) \Rightarrow \varphi_{e}{ }^{1}\left(\varphi_{d}^{1}\left(p_{\alpha}(n)\right)\right) \in F$. Thus, $\varphi_{e}{ }^{1}$ maps elements of $\varphi_{d}^{1}(\alpha)$ to smaller elements of $\varphi_{d}{ }^{1}(\alpha)$ with at most finitely many exceptions. Therefore $\varphi_{d}{ }^{1}(\alpha)$ is retraced by some finite modification, $\varphi_{e^{\prime}}{ }^{1}$ of $\varphi_{e}{ }^{1}$. But, as already noted, $\varphi_{d}^{1}\left(p_{\alpha}(m)\right)>\varphi_{d}^{1}\left(p_{\alpha}(n)\right)$ whenever $m>n \geqq d$. Hence there exists a number $n_{0} \geqq d$ such that $\quad n>n_{0} \Rightarrow \varphi_{e^{\prime}}{ }^{1}\left(\varphi_{d}^{1}\left(p_{\alpha}(n)\right)\right)=\varphi_{d}^{1}\left(p_{\alpha}(n-1)\right)$. Taking $n>$ $\max \left\{n_{0}, k\right\}$, then, we have: $\varphi_{k}^{1}\left(\varphi_{e^{\prime}}^{1}\left(\varphi_{d}^{1}\left(p_{\alpha}(n+1)\right)\right)\right)=\varphi_{k}^{1}\left(\varphi_{d}^{1}\left(p_{\alpha}(n)\right)\right)$ 
$<\varphi_{d}^{1}\left(p_{\alpha}(n+1)\right)$. It is thus proven that $\varphi_{d}{ }^{1}(\alpha)$ is, in fact, $t$-retraceable. To get the last assertion in the statement of Theorem 3.4 as a special case of what has just been proved, simply note that if $T_{0}-3+T_{1}$ and $\tau_{1}$ is an infinite co-r.e. branch of $T_{1}$, then $\tau_{0}$ is also co-r.e. where $\tau_{0}=$ the unique infinite branch $\tau$ of $T_{0}$ such that $\tau \subseteq \tau_{1}$.

As a second application, we get a virtually instantaneous proof of a stronger version of [13, Lemma 4.21] (the proof of which was merely sketched in [13]).

3.5 Theorem. Let $T_{1}$ be a non-trivial semicomputable tree. Then there exists a semicomputable tree $T_{0}$, defined by a partial recursive function $p_{0}$, such that $T_{0} 3_{+} T_{1} \& T_{0}$ is finite-branching if $T_{1}$ is finitebranching \& $(\forall \tau)\left[\left(\tau=\right.\right.$ an infinite branch of $\left.T_{0}\right) \Rightarrow\left(p_{0}\right.$ retraces $\tau \&$ $\left.\left.\tau \geqq 0^{\prime}\right)\right]$.

Proof. Applying Theorem 3.1, let $T_{0}$ be a uniformly thin-branched tree such that $T_{0}-3_{+} T_{1} \& T_{0}$ is finite-branching provided $T_{1}$ is. Then each infinite branch $\tau$ of $T_{0}$ is retraced by $p_{0}$, where $p_{0}$ is the partial recursive function defining $T_{0}$. Moreover, each such $\tau$ has property (1) of Theorem 3.2; i.e., if $p_{\tau}$ is the function which enumerates $\tau$ in order of magnitude then $p_{\tau}$ eventually bounds any given partial recursive function. But this latter property is well known to imply that $\tau \geqq 0^{1}$, and the proof is complete.

It is natural to inquire whether $t$-retraceability is an isolic invariant; i.e., whether $[\alpha] t$-retracable $\Rightarrow \alpha t$-retraceable. Since obviously any $t$-retraceable set is hyperimmune, the following theorem shows that the answer is in the negative.

3.6 ThEOREM. There exists a nonrecursive, recursively enumerable set $W$ and a 1-1 partial recursive function $p$ such that $N-W$ is a $t$ retraceable set $\& N-W \subseteq \delta p \& p(N-W)$ is not hyperimmune.

Proof. The proof is a virtual repetition of the proof of [16, Theorem 1]; so, since it is possible to prove a stronger theorem (see Theorem 3.7 below), we shall merely outline the procedure. We replace Lemma 1 of [16] by the following Lemma $1^{\prime}$. There exists a co-infinite r.e. set $\alpha$ having the following property: $N-\alpha$ is $t$ retraced by a partial recursive function $f$ such that there is a total recursive function $r$ satisfying $(\forall n)[r(n)>\operatorname{card}(\{x \mid x \in \delta f \&$ $\left.\left.\left.f^{*}(x)=n\right\}\right)\right]$. The proof of this modified version of [16, Lemma 1] proceeds in exactly the same way as the proof of [16, Lemma 1] itself, except that in the construction we move markers so as to secure $t$-retraceability (of the set of final marker-positions) rather than mere eventual bounding of arbitrary partial recursive functions; 
it remains easy to display a recursive bound $r(m)$ on the total number of different positions held by the marker $\Lambda_{m}$ during the course of the construction. Having proved Lemma 1 ', we finish by repeating verbatim the argument for [16, Theorem 1] given on $p .83$ of [16].

By suitably combining the constructions of [15] and [16, proof of Theorem 1], we can establish the following stronger result:

3.7 TheOREM. There exists a nonrecursive recursively enumerable set $W$ and a 1-1 partial recursive function $p$ such that $N-W$ is $t$ retraceable \& $N-W \subseteq \delta p \& p(N-W)$ is neither retraceable nor hyperimmune.

We shall omit the proof of Theorem 3.7, since it is both cumbersome and ad hoc.

In conclusion, as further illustrations of the uses of Theorem 2.2, we state without proof three theorems (representing refinements of known results) which are simple corollaries to Theorems 3.1 and 3.2. (For the definition of the relation $\varliminf^{*}$ mentioned in 3.9 , see [2], [4], or [10]; as Barback has shown in [4], $\varliminf^{*}$ restricted to $\Lambda_{R} \times \Lambda_{R}$ coincides with $\left.\Lambda_{\leqq}\right|_{\Lambda_{R} \times \Lambda_{R}}$, where $\Lambda_{\leqq}$is the canonical extension to isols of the ordinary $\leqq$ relation in $N \times N$.)

3.8 Theorem. If $A \in \Lambda_{R}{ }^{\infty}$ and $\alpha \in A$, then there is a $\Pi_{1}{ }^{0}$ set $\beta$ such that $[\alpha \cap \beta] \in \Lambda_{R}{ }^{\infty} \&[\alpha \cap \beta]$ is multiple-free ([8]); whence $[\alpha \cap \beta]$ is neither an odd nor an even isol; whence (see [10] for a discussion of the $\Phi_{f^{-}}$operators and their connection with idempotency) the isols $\Phi_{2 n}([\alpha \cap \beta])$ and $\Phi_{2 n+1}([\alpha \cap \beta])$ have non-trivial idempotent difference in $\Lambda_{R}{ }^{*}$.

3.9 Theorem. Let $A \in \Lambda_{R}{ }^{\infty}, \alpha \in A$. Then there exist sets $\beta, \gamma$, and $\tau$ such that $\boldsymbol{\beta}$ is $\Pi_{1}{ }^{0} \&\{[\gamma],[\tau]\} \subseteq \Lambda_{R}{ }^{\infty} \&[\alpha \cap \beta]=[\gamma]+$ $\left.[\tau] \&[\gamma] \not\right|^{*}[\tau] \&[\tau] \neq^{*}[\gamma]$. (In the co-simple case, if $\alpha$ is a retraceable $\Pi_{1}{ }^{0}$ set having degree a where $a^{1}=0^{11}$, then we can require $\beta=\gamma=\tau=\mathrm{a}$; this, however, follows not from Theorem 3.1 but from a variant of Theorem 2.2.

3.10 TheOREM (refining to the co-simple case an improvement due to Gersting of a theorem of Hassett). There exist isols $A \in \Lambda_{Z R}^{\infty}$ and $B \in \Lambda_{Z R}^{\infty}$ such that $\boldsymbol{B}$ is an isolic summand of $\boldsymbol{A}$ but $\Phi_{f}(\boldsymbol{A}) \varliminf^{*}$ $B$ fails for all strictly increasing recursive functions $f$.

We remark that an even stronger result than Theorem 3.10 is obtainable via the techniques of the present paper: if a is a given r.e. degree satisfying $\boldsymbol{a}^{1}=\mathbf{0}^{11}$ and $\gamma$ is a $\Pi_{1}{ }^{0}$ regressive set of degree $\boldsymbol{a}$, then, in Theorem 3.10, we can require that $\boldsymbol{A}$ be a multiple-free 
isol represented by a $\Pi_{1}{ }^{0}$ subset $\alpha$ of $\gamma$ such that $\boldsymbol{\alpha}=\mathbf{a}$. (Note that this simultaneously strengthens 3.8 in the co-simple case.)

4. Relativization. It is easily seen that the results of the preceding sections can be relativized routinely to non-semicomputable trees. For instance, when thus relativized Theorem 2.2 becomes

THeOrem 2.2*. Let $d$ be any fixed degree of unsolvability; and let $T_{1}$ be a non-trivial tree whose defining function $p_{1}$ is partial recursive in $d$. Let $g$ be a function from $N$ into $N$ such that $g$ is $\Delta_{2}$ in $d$. Then, there exists a tree $T_{0}$ (finite-branching if $T_{1}$ is) such that

the function $p_{0}$ whose graph is $T_{0}$ is partial recursive in $d$;

$$
T_{0} \rightarrow T_{1}
$$

$(\exists n)\left(\forall \alpha_{0}\right)\left(\forall \alpha_{1}\right)\left[\left(\alpha_{0}=\right.\right.$ an infinite branch of $T_{0} \& \alpha_{1}$ $=$ an infinite branch of $\left.T_{1} \& \alpha_{0} \subseteq \alpha_{1}\right) \Rightarrow \alpha_{1}-\alpha_{0}=$ $\left.\alpha_{1} \cap W_{n}^{d}\right]$, where $W_{n}^{d}=$ the $n$-th set recursively enumerable in $d$; and

$T_{0}$ is g-dominant.

\section{REFERENCES}

1. K. I. Appel and T. G. McLaughlin, On properties of regressive sets, Trans. Amer. Math. Soc. 115 (1965), 83-93. 420.

2. J. Barback, Two notes on regressive isols, Pacific J. Math. 16 (1966), 407 -

3. - Regressive upper bounds, Seminario Matematico 39 (1967), 248-272.

4. - Two notes on recursive functions and regressive isols, Trans. Amer. Math. Soc. 144 (1969) 77-94.

5. —- Extensions to regressive isols, Math. Scand. 25 (1969), 159-177.

6. J. Barback and W. D. Jackson, Representations as infinite series of isols, Compositio Math. 22 (1970), 347-365.

7. J. C. E. Dekker and J. Myhill, Retraceable sets, Canadian J. Math. 10 (1958), $357-373$

8. - Recursive equivalence types, University California Publ. Math. (N.S) 3 (1960), 67-214.

9. J. C. E. Dekker, Infinite series of isols, Proc. Symposia Pure Math., Amer. Math. Soc. 5 (1962), 77-96.

10. — Regressive isols, Sets, Models and Recursion Theory, North-Holland Publ. Co., Amsterdam, 1967, 272-296.

11. E. Ellentuck, Universal cosimple isols, Pacific J. Math 42 (1972), 629-638.

12. M. J. Hassett, A mapping property of regressive isols, Illinois J. Math. 14 (1970), 478-487.

13. C. G. Jockusch, Jr. and T. G. McLaughlin, Countable retracing functions and $\mathrm{II}_{2}{ }^{0}$ predicates, Pacific J. Math., 30 (1969), 67-93. 
14. T. G. McLaughlin, Hereditarily retraceable isols, Bull. Amer. Math. Soc. 73 (1967), 113-115.

15. — Retraceable sets and recursive permutations, Proc. Amer. Math. Soc. 17 (1966), 427-429.

16. - Some counterexamples in the theory of regressive sets, Zeitschrift $f$. Math. Logik und Grundl. d. Math. 13 (1967), 81-87.

17. F. J. Sansone, The backward and forward summation of infinite series of isols, Math. Scand. 24 (1969), 217-220.

18. J. R. Shoenfield, On degrees of unsolvability, Annals of Math. 69 (1959), 644-653.

19. C. E. M. Yates, Recursively enumerable sets and retracing functions, Zeitschrift f. Math. Logik und Grundl. d. Math. 8 (1962), 331-345.

20. - Three theorems on the degrees of recursively enumerable sets, Duke Math. J. 32 (1965), 461-468.

UNIVERSITY OF IllinoIs, URBaNa, IllinoIs 61803, AND

TeXas Tech University, LubBock, TeXas 79409 